\title{
Antimicrobial susceptibility and body site distribution of community isolates of Coagulase
}

\section{Negative Staphylococci}

\author{
Jorunn Pauline Cavanagh ${ }^{* 1,2}$, Runa Wolden ${ }^{1,2}$, Philipp Heise ${ }^{3}$, Eirin Esaiassen ${ }^{1,2}$, Claus Klingenberg ${ }^{1,2}$ and Elizabeth G. Aarag
} Fredheim ${ }^{4}$

${ }^{1}$ Department of Paediatrics, University Hospital of North Norway, Troms $\varnothing$, Norway

${ }^{2}$ Department of Clinical Medicine, UiT The Arctic University of Norway, Troms $\varnothing$, Norway

${ }^{3}$ Institute of Microbiology and Molecular Biology, University of Giessen, Giessen, Germany

${ }^{4}$ Department of Pharmacy, UiT The Arctic University of Norway, Troms $\varnothing$, Norway

*Corresponding author; Jorunn Pauline Cavanagh, Paediatric Research Group, University Hospital of North Norway 9037. Tromsø,

Norway. Phone: + 47 77646950. E-mail: pauline.cavanagh@uit.no 


\section{Abstract}

16 The primary aim of this study was to determine antimicrobial resistance in coagulase negative staphylococci (CoNS) from healthy

17 adults in the community. Healthy adults $(\mathrm{n}=114)$ were swabbed on six body sites; both armpits, both knee pits and both sides of the

18 groin. Species determination was performed using MALDI-TOF and susceptibility testing for eleven relevant antimicrobials was

19 performed by the disc diffusion method and minimal inhibitory concentration gradient test.

20 In total, 693 CoNS-isolates were identified. Susceptibility testing was done on 386 isolates; one CoNS from each species found on 21 each participant from the different body sites. The prevalence of antimicrobial resistance in the CoNS isolates were; erythromycin

22 (24.6\%), fusidic acid (19.9\%), tetracycline (11.4\%), clindamycin (7.8\%), gentamicin (6.2\%) and cefoxitin (4.1\%). Multidrug resistance

23 was observed in 5.7\% of the isolates. Staphylococcus epidermidis and S. hominis were the first and second most prevalent species on

24 all three body sites. We conclude that CoNS isolates from healthy adults in the community have a much lower prevalence of

25 antimicrobial resistance than reported in nosocomial CoNS isolates. Still, we believe that levels of resistance in community CoNS

26 should be monitored as the consumption of antimicrobials in primary care in Norway is increasing.

27

28 Running head: Antimicrobial susceptibility of community coagulase negative staphylococci .

29 Keywords: Coagulase negative staphylococci, commensals, body site distribution, antimicrobial resistance, body site distribution, community CoNS 


\section{INTRODUCTION}

33 The development and global spread of antimicrobial resistance is a threat to modern medicine. The commensal skin flora, dominated

34 by coagulase negative staphylococci (CoNS), may act as a reservoir of antimicrobial resistance, and transfer resistance genes to more

35 virulent staphylococci such as Staphylococcus aureus (1-4). Over the last decades, CoNS have received increased interest as important

36 opportunistic nosocomial pathogens frequently involved in medical implant infections and infections in immunocompromised patients,

37 e.g. patients with haematological diseases and very preterm infants (5). Studies on antimicrobial resistance in CoNS have mainly

38 focused on invasive isolates, commonly from hospitalized patients (6-8). Multidrug-resistant hospital adapted clones have been

39 identified in both $S$. epidermidis and S. haemolyticus $(6,9)$. However, only limited data exist regarding antimicrobial resistance among

40 community CoNS isolates (10-12). Furthermore, CoNS species with different resistance and virulence traits may have different niches

41 on the human body (2).

42

43 The primary aim of this study was to determine susceptibility to commonly used antimicrobial agents in a selection of CoNS isolates

44 from healthy adults in the community. Secondly, we report the body site distribution of CoNS on three body sites screened in this study. This may increase our understanding of the role CoNS play as reservoirs of antimicrobial resistance. 


\section{MATERIAL AND METHODS}

Healthy adult volunteers (age 18-49 years, mean age; 25.5 years) were recruited, primarily from different sport teams $($ basketball, $\mathrm{n}=$ 14, ice hockey, $n=9$, four different football teams $n=55$ and members of a student's sports centre $n=19)$ and office employees ( $n=17$ ). All participants filled in a questionnaire regarding antimicrobial consumption, hospitalization and travel abroad during the last three months. Health care workers and volunteers who reported antimicrobial consumption and/or contact with health care institutions during the last three months were not included in the study.

All participants were swabbed with Amies charcoal transport swabs (Sarstedt, Nümbrecht, Germany) on six body sites; both armpits, both knee pits and both sides of the groin. Swabs were streaked on blood agar plates (Oxoid, Basingstoke, England) and incubated overnight (16-20 hours) at $37^{\circ} \mathrm{C}$. All visible CoNS with different morphotypes on blood agar plates were selected for further analyses (5-36 colonies from each participant). The phenotypes were characterized by colonies of different diameter with white, grey, creamy or yellow pigmentation and moderately heavy, weak or absent haemolysis.

Species determination was performed with MALDI-TOF MS using a Microflex LT instrument (Bruker Daltonics, Massachusetts, USA), Flexcontrol software and the Biotyper database (Bruker Daltonics, Massachusetts, USA) (13). A simple extraction method with $70 \%$ formic acid (Sigma-Aldrich, St. Louis, MO, USA) was used on the isolates before adding HCCA matrix solution (Bruker Daltonics, Massachusetts, USA / Sigma-Aldrich, St. Louis, MO, USA). Both positive (ATCC 9144 S. aureus) and negative (matrix 
66

67

68

69

70

71

72

73

74

75

76

77

78

\section{Ethical approval}

80

\section{Ethical approval}

solution) controls were applied on each test plate run on the MALDI-TOF MS. All samples were run in parallel. Processing of samples were done according to the user manual (13) Only samples that obtained a log (score) value of $\geq 2$ were used further, as these results are considered to give a high probability of identification at the species level (14).

After species determination, one CoNS-isolate of each species was randomly selected from each participant, and underwent testing for antimicrobial susceptibility. Antimicrobial susceptibility testing and interpretation was performed according to EUCAST guidelines (15). Oxoid MH agar plates were used (Oxoid, Basingstoke, England).The disk diffusion test was used for cefoxitin (as a marker for methicillin resistance), trimethoprim-sulfamethoxazole (TMS), clindamycin, erythromycin, fusidic acid, gentamicin, linezolid, tetracycline, ciprofloxacin and rifampicin (Oxoid, Basingstoke, England). A minimal inhibitory concentration (MIC) gradient test was used for vancomycin susceptibility testing of all isolates, and for selected isolates with linezolid inhibition zones around defined breakpoints. (Liofilchem, Roseto degli Abruzzi, Italy). ATCC 29213 S. aureus was used as reference strain. All isolates were also tested for inducible resistance to clindamycin (15). Multidrug resistance (MDR) was defined as resistance to at least three classes of antimicrobial agents.

The Regional Committee (REC) for Medical Research Ethics
Informed written consent was obtained from all participants. 


\section{RESULTS}

In total, 114 participants (57 male and 57 female) were included in the study. None of the participants had consumed any antimicrobial agents, worked at, or been admitted to a health care institution 3 months prior to the swabbing. A total of 693 CoNS were identified from the different body sites of the 114 volunteers (Figure 1). Eleven potential Staphylococcus species were not included because of a $\log$ (score) value < 2 on MALDI-TOF MS. S. epidermidis and S. hominis were the first and second most prevalent species at all three body sites, body site distribution and prevalence is listed in Figure 1.

We performed antimicrobial susceptibility testing on 386 isolates; one CoNS from each species found on each participant (Table 1). Different CoNS species per person included in the antimicrobial susceptibility testing varied from one to seven (mean=3). In total 110 S. epidermidis, 93 S. hominis, 59 S. capitis, 48 S. haemolyticus, 38 S. lugdunensis, 13 S. saprophyticus, and 25 other CoNS were tested. The highest prevalence of resistance was towards erythromycin 95/386 (24.6\%), fusidic acid 77/386 (19.9\%) and tetracycline 44/386 $(11.4 \%)$. There was a very low prevalence $(<2 \%)$ of resistance towards rifampicin, ciprofloxacin and TMS. Overall, $16 / 386(4.1 \%)$ of CoNS isolates were methicillin resistant. Resistance to vancomycin or linezolid was not detected in any isolates. MDR was observed in $5.2 \%$ of the isolates. S. hominis displayed the highest prevalence of MDR (10.8\%), followed by S. epidermidis (6.4\%) and S. haemolyticus $(6.3 \%)$. In $16.6 \%$ of the participants, all of the tested strains were susceptible to all antimicrobial agents. In $13.5 \%$ of the participants, all strains tested displayed resistance to one or more antimicrobial agents. Resistant isolates were not associated with any specific body sites. 
101 There was no correlation between the prevalence of antimicrobial resistance and participants belonging to different sports teams, nor

102 was there any differences observed in prevalence of antimicrobial resistance in the different age groups or between the male and

103 female participants (data not shown).

104

105

\section{DISCUSSION}

107 This is, to our knowledge, the largest, recent study focussing on antimicrobial susceptibility in community CoNS. The commensal

108 CoNS isolates displayed resistance to all antimicrobial classes tested apart from vancomycin and linezolid. MDR was detected in 5.2\%

109 of the isolates. However, in around 1 of 6 participants no antimicrobial resistant CoNS-isolates were found. The highest prevalence of

110 antimicrobial resistance was towards erythromycin, fusidic acid, tetracycline and clindamycin, all antimicrobial agents commonly

111 prescribed in primary health care to treat respiratory tract and skin infections (16). A recent Portuguese study on community CoNS

112 reported overall higher prevalence of resistance than in our Norwegian isolates, and showed a higher prevalence of resistance towards

113 agents commonly prescribed antibiotics in primary care (12). Similar rates of antibiotic resistance have also been reported in

114 community isolates of $S$. epidermidis, $(11,17,7,18)$. It has previously been demonstrated that CoNS skin commensals easily develop

115 resistance towards ciprofloxacin and betalactams, due to secretion of these antimicrobial agents in sweat, reflecting the ability to 
116 rapidly adapt to changing external pressure $(19,20)$. However, the rates of resistance to cefoxitin and ciprofoxacin was low in our

117 study.

118

119 In Norway, around $85 \%$ of the total human consumption of antimicrobial agents is in the primary care setting. The three most

120 commonly prescribed groups of antibiotics are penicillins, tetracyclins and macrolides $(21,22)$, the consumption in Troms county is

121 marginally lower that at the national level (personal communication, Hege Blix, Norwegian Institute of Public Health). The high

122 consumption of macrolides in Norway may explain the relatively high prevalence of macrolide resistance among community CoNS

123 isolates (22). In the Norwegian national guidelines for antibiotic use in primary care, macrolides are not recommended as first-line

124 therapy for any other conditions than pneumonia caused by mycoplasma and/or chlamydophilia (23), but the relatively high consumption indicates that guidelines are not universally followed. Overuse of macrolides may contribute to increased antibiotic resistance (24), and the current macrolide use in Norway is higher than wanted by the regulatory authorities. patterns of clinical CoNS isolates from total hip arthroplasty infections during 1993-2007 reported an increase in methicillin resistance rates from 57 to $84 \%$, as well as increasing rates of resistance to most other antimicrobials tested (26). Antimicrobial resistance is, however, not routinely monitored in commensal CoNS and we do not know if the prevalence of resistance in the community has increased. Compared to community isolates, clinical isolates have a much higher prevalence of antimicrobial resistance, most likely 
133 reflecting that hospital adapted resistant clones seem to outcompete the commensal flora (9). Only $5.2 \%$ of the community CoNS in

134 our study displayed MDR, but these isolates may also have a competitive advantage if entering the hospital and being exposed to the 135 increased antimicrobial pressure in the hospital setting. Interestingly we observed that $13.5 \%$ of the participants were colonised with

136 isolates that were resistant to one or more antimicrobial agents. These individuals might act as a reservoir of antimicrobial resistance

137 genes in the community to other CoNS or S. aureus. Acquisition of antimicrobial resistance genes by horizontal gene transfer between

138 closely related staphylococcal species has been hypothesised as the main cause for the successful spread of the community associated

139 USA 300 methicillin-resistant S. aureus clone $(27,28)$.

140

141 Selection of swab sites for collection of strains was based on previously reported body sites frequently colonised with CoNS; the

142 axillae, the groin and a the more dry extremities such as the knee $(29,30)$. As expected, S. epidermidis was the dominant species on all

143 body sites. The second most common species was $S$. hominis, previously reported to commonly colonize the axillae, arms and legs and

144 areas with apocrine glands such as the inguinal and perineal areas $(31,32,29)$. Of note is that $S$. capitis, previously thought to be most

145 prevalent on the head, was frequently found in the samples from the groin and the knee pit, whereas S. saprophyticus, a urinary tract

146 pathogen, was rarely found in the groin (2). 
148 This study has strengths and limitations. We took care to ensure that the isolates were truly community isolates by not including

149 volunteers who recently had been treated with antimicrobial agents or were working in health care facilities. Due to a large number of

150 isolates we decided to restrict susceptibility testing to one isolate of each species from each participant. Spread of community acquired

151 methicillin resistant $S$. aureus between members of sports teams in close contact sports, such as football has been demonstrated (33,

152 34). As we have swabbed groups of participants belonging to the same sports teams, we might have introduced a potential bias due to a

153 possible spread of strains between members of the same sports teams, carrying specific antimicrobial resistance genes. This could

154 artificially increase the prevalence of antimicrobial resistance in our collection, compared to the general population. However, we

155 believe that the large number of isolates included to a large extent reflect the antimicrobial susceptibility pattern of CoNS outside

156 hospitals in Norway. Our data on body site distribution clearly show that different CoNS species may have other body niches than

157 previously reported $(29,30)$ We did not perform susceptibility testing on all 693 isolates detected from all body sites. Thus, we cannot

158 specify resistance pattern to each body site.

159 There is a paucity of information regarding antimicrobial resistance in commensal CoNS. We conclude that the prevalence of

160 antimicrobial resistance among community CoNS in Norway is relatively low. However, MDR is present and these isolates may be

161 more adaptable when introduced in a hospital setting. With the increase in antimicrobial prescriptions in primary care in Norway (22),

162 prevalence of resistance in community CoNS should be monitored. Further comparative studies should be conducted in order to

163 understand which factors are involved in hospital adaption of community isolates resulting in the high prevalence of MDR-CoNS in

164 hospitals. 


\section{FUNDING}

167 This work was supported by The Northern Norway Regional Health Authority.

168

\section{TRANSPARENCY DECLARATIONS}

170 The authors have no interests to declare.

\section{AUTHOR'S CONTRIBUTION}

173 JPC participated in conception and design, collection of strains, antimicrobial susceptibility testing, interpretation of data and writing 174 of the manuscript.

175 RW participated in collection of strains, antimicrobial susceptibility testing, MALDI-TOF MS, interpretation of data and manuscript

176 writing.

$177 \mathrm{PH}$ participated in antimicrobial susceptibility testing and manuscript writing.

178 EE participated in antimicrobial susceptibility testing and manuscript writing.

179 CK participated in conception, design and writing the manuscript. 
180 EGAF participated in conception and design, collection of strains, writing of the manuscript and given final approval of the manuscript

181 to be published.

182 All authors read and approved the final manuscript.

183

184 Acknowledgements

185 We would like to thank all study participants.

186

187

188

189

190

191

192

193

194

195 


\section{REFERENCES}

199 1. Otto M. Coagulase-negative staphylococci as reservoirs of genes facilitating MRSA infection. BioEssays. 2013;35(1):4-11.

200 2. von Eiff C, Peters G, Heilmann C. Pathogenesis of infections due to coagulase-negative staphylococci. Lancet Infect Dis.

$201 \quad 2002 ; 2: 677-85$.

202 3. Archer GL, Johnston JL. Self-transmissible plasmids in staphylococci that encode resistance to aminoglycosides. Antimicrob Agents

203 Chemother. 1983;24(1):70-7.

204 4. Winstel V, Liang C, Sanchez-Carballo P, Steglich M, Munar M, Bröker BM et al. Wall teichoic acid structure governs horizontal 205 gene transfer between major bacterial pathogens. Nature Communications. 2013;4:2345.

206 5. Nouri LBZ, Caitriona MG, Fitzgerald JR. Pathogenomics of the staphylococci: insights into niche adaptation and the emergence of 207 new virulent strains. FEMS Microbiology Letters. 2008;289(1):1-12.

208 6. Cavanagh JP, Hjerde E, Holden MTG, Kahlke T, Klingenberg C, Flægstad T et al. Whole-genome sequencing reveals clonal 209 expansion of multiresistant Staphylococcus haemolyticus in European hospitals. Journal of Antimicrobial Chemotherapy.

210 2014;69(11):2920-7.

211 7. Cherifi S, Byl B, Deplano A, Nonhoff C, Denis O, Hallin M. Comparative Epidemiology of Staphylococcus epidermidis Isolates 212 from Patients with Catheter-Related Bacteremia and from Healthy Volunteers. Journal of Clinical Microbiology. 2013;51(5):1541-7.

213 8. Venkatesh MP, Placencia F, Weisman LE. Coagulase-Negative Staphylococcal Infections in the Neonate and Child: An Update. 214 Seminars in Pediatric Infectious Diseases. 2006;17(3):120-7.

215 9. Widerström M, Wiström J, Sjöstedt A, Monsen T. Coagulase-negative staphylococci: update on the molecular epidemiology and 216 clinical presentation, with a focus onStaphylococcus epidermidis and Staphylococcus saprophyticus. European Journal of Clinical 217 Microbiology \& Infectious Diseases. 2012;31(1):7-20.

218 10. Larson EL, McGinley KJ, Foglia AR, Talbot GH, Leyden JJ. Composition and antimicrobic resistance of skin flora in hospitalized 219 and healthy adults. Journal of Clinical Microbiology. 1986;23(3):604-8. undergoing a primary hip- or knee replacement in Southern Sweden. Acta Orthopaedica. 2013;84(1):87-91. 12. Oliveira F, Cerca N. Antibiotic resistance and biofilm formation ability among coagulase-negative staphylococci in healthy individuals from Portugal. J Antibiot. 2013;66(12):739-41.

224 13. Bruker Daltonics M, USA. MALDI Biotyper 3.1 User Manual. 2012. 
14. Szabados F, Woloszyn J, Richter C, Kaase M, Gatermann S. Identification of molecularly defined Staphylococcus aureus strains using matrix-assisted laser desorption/ionization time of flight mass spectrometry and the Biotyper 2.0 database. Journal of Medical Microbiology. 2010;59(7):787-90.

15. EUCAST. Breakpoint tables for interpretation of MICs and zone diameters v 5.0. In: The European Committee on Antimicrobial Susceptibility Testing. EUCAST. 2015. http://www.eucast.org/clinical_breakpoints/. Accessed May 292015.

16. van Bijnen EME, Paget WJ, den Heijer CDJ, Stobberingh EE, Bruggeman CA, Schellevis FG et al. Primary care treatment guidelines for skin infections in Europe: congruence with antimicrobial resistance found in commensal Staphylococcus aureus in the community. BMC Family Practice. 2014;15(1).

17. Rolo J, de Lencastre H, Miragaia M. Strategies of adaptation of Staphylococcus epidermidis to hospital and community: amplification and diversification of SCCmec. Journal of Antimicrobial Chemotherapy. 2012;67(6):1333-41.

18. Widerstrøm M, Wistrøm J, Ek E, Edebro H, Monsen T. Near absence of methicillin-resistance and pronounced genetic diversity among Staphylococcus epidermidis isolated from healthy persons in northern Sweden. APMIS. 2011;119(8):505-12.

19. Høiby N, Pers C, Johansen HK, Hansen H. Excretion of $\beta$-Lactam Antibiotics in Sweat - a Neglected Mechanism for Development of Antibiotic Resistance? Antimicrobial Agents and Chemotherapy. 2000;44(10):2855-7.

20. Høiby N, Jarløv JO, Kemp M, Tvede M, Bangsborg JM, Kjerulf A et al. Excretion of ciprofloxacin in sweat and multiresistant Staphylococcus epidermidis. The Lancet. 1997;349(9046):167-9.

21.NORM/NORM-VET 2014. Usage of Antimicrobial Agents and Occurrence of Antimicrobial Resistance in Norway. Troms $\varnothing$ / Oslo 2015. ISSN:1502-2307 (print) / 1890-9965 (electronic).

22. European Centre for Disease Prevention and Control. Trend of antimicrobial consumption by country ECDC. 2015.

http://ecdc.europa.eu/en/healthtopics/antimicrobial_resistance/esac-net-database/Pages/trend-consumption-by-country.aspx. Accessed 10.03.2015 2015.

23. National guidelines for antibiotic use in ambulatory care and nursing homes. Published 2012 by The Norwegian Directorate of Health. The Norwegian Directorate of Health. 2012. https://helsedirektoratet.no/retningslinjer/nasjonal-faglig-retningslinje-forantibiotikabruk-i-primerhelsetjenesten. Accessed 06.06.2016.

24. Bergman M, Huikko S, Pihlajamäki M, Laippala P, Palva E, Huovinen P et al. Effect of Macrolide Consumption on Erythromycin Resistance in Streptococcus pyogenes in Finland in 1997-2001. Clinical Infectious Diseases. 2004;38(9) 1251-1256.

25. Keim LS, Torres-Filho SR, Silva PV, Teixeira LA. Prevalence, Aetiology and Antibiotic Resistance Profiles of Coagulase Negative Staphylococci Isolated in a Teaching Hospital. Brazilian Journal of Microbiology. 2011;42(1):248-55.

26. Lutro O, Langvatn H, Dale H, Schrama JC, Hallan G, Espehaug B et al. Increasing Resistance of Coagulase-Negative Staphylococci in Total Hip Arthroplasty Infections: 278 THA-Revisions due to Infection Reported to the Norwegian Arthroplasty Register from 1993 to 2007. Advances in Orthopedics. 2014;2014:7.

27. Knox J, Uhlemann A-C, Lowy FD. Staphylococcus aureus infections: transmission within households and the community. Trends in Microbiology. 2015 23(7):437-44. 
258 28. Alam MT, Read TD, Petit RA, Boyle-Vavra S, Miller LG, Eells SJ et al. Transmission and Microevolution of USA300 MRSA in 259 U.S. Households: Evidence from Whole-Genome Sequencing. mBio. 2015;6(2).

260 29. Kloos WE, Bannerman TL. Update on clinical significance of coagulase-negative staphylococci. Clinical Microbiology Reviews. $261 \quad 1994 ; 7(1): 117-40$.

262 30. Becker K, Heilmann C, Peters G. Coagulase-Negative Staphylococci. Clinical Microbiology Reviews. 2014;27(4):870-926.

263 31. Grice EA, Segre JA. The skin microbiome. Nat Rev Micro. 2011;9(4):244-53.

264 32. Kloos WE, Musselwhite MS. Distribution and Persistence of Staphylococcus and Micrococcus Species and Other Aerobic Bacteria 265 on Human Skin. Applied Microbiology. 1975;30(3):381-95.

266 33. Kurkowski C. CA-MRSA. The new sports pathogen. Orthopedic nursing. 2007;26(5):310-4.

267 34. Dao MN, Laurene M, Elizabeth B. Recurring Methicillin-resistant Staphylococcus aureus Infections in a Football Team. Emerging 268 Infectious Disease journal. 2005;11(4):526.

269

270

271

272

273

274

275

276

277 


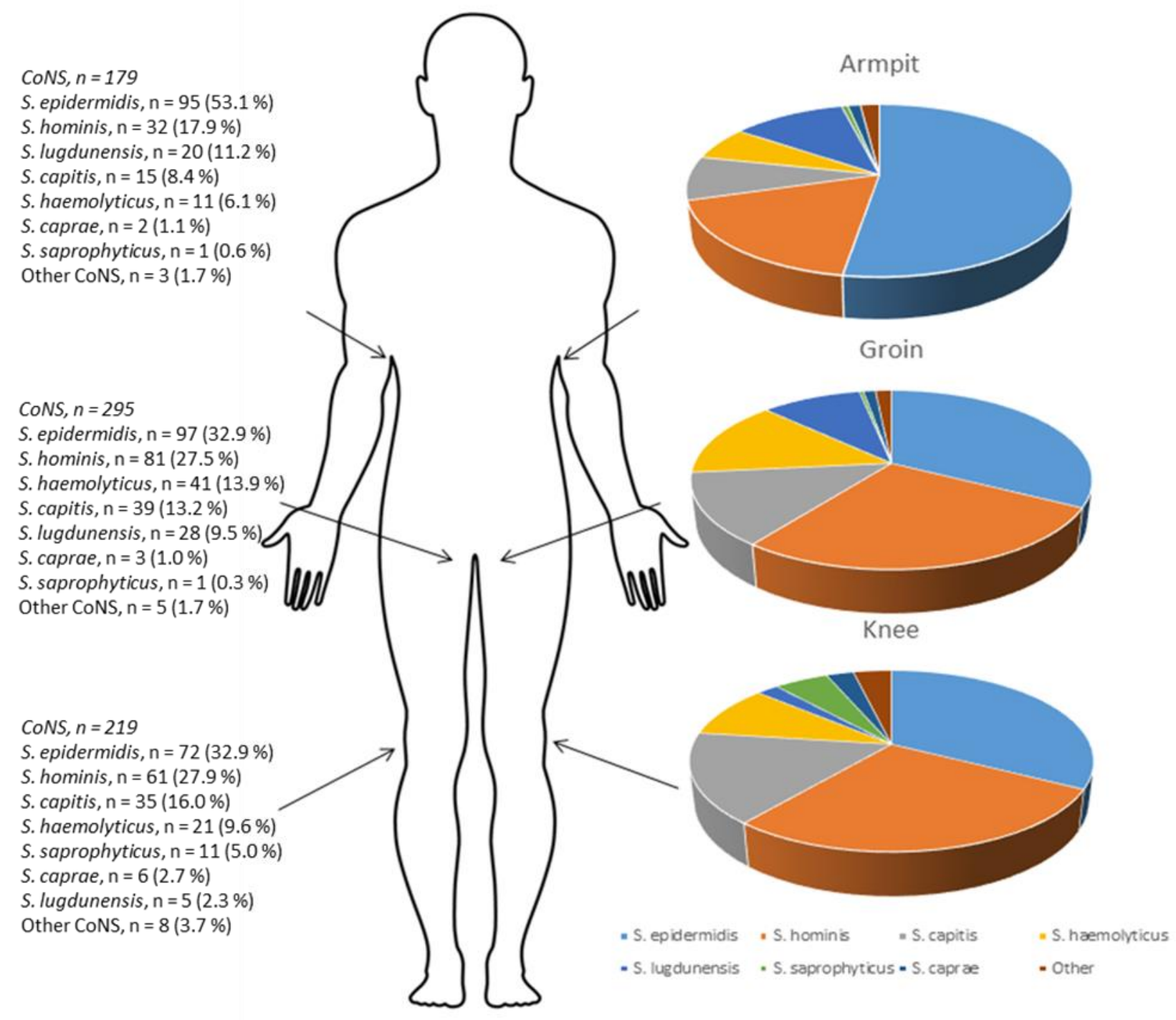

Figure 1: Body site distribution of CoNS, the prevalence represent the proportion of CoNS species found at each body site for 114 
282 Table 1: Prevalence of antimicrobial resistance (\%) in 386 community CoNS isolates from healthy adults.

283

\begin{tabular}{llllllll}
\hline N Cefoxitin & TMS & Clindamycin & Erythromycin & Fusidic & Gentamicin & Tetracycline Ciprofloxacin Rifampicin MDR*
\end{tabular}

acid

\begin{tabular}{|c|c|c|c|c|c|c|c|c|c|c|c|}
\hline S. epidermidis & 110 & $2(1.8)$ & $2(1.8)$ & $8(7.3)$ & $29(26.4)$ & $29(26.4)$ & $11(10.0)$ & $6(5.5)$ & 0 & $1(0.9)$ & $5(4.5)$ \\
\hline S. hominis & 93 & $6(6.5)$ & $2(2.2)$ & $11(11.8)$ & $32(34.4)$ & $29(31.2)$ & $7(7.5)$ & $28(30.1)$ & $1(1.1)$ & 0 & $10(10.8)$ \\
\hline S. capitis & 59 & $3(5.1)$ & 0 & $4(6.8)$ & $2(3.4)$ & $6(10.2)$ & $3(5.1)$ & $4(6.8)$ & $2(3.4)$ & 0 & $2(3.4)$ \\
\hline S. haemolyticus & 48 & $3(6.3)$ & 0 & 7 (14.6) & $29(60.4)$ & $6(12.5)$ & $1(2.1)$ & $4(8.3)$ & $2(4.2)$ & 0 & $3(6.3)$ \\
\hline S. lugdunensis & 38 & 0 & 0 & 0 & $1(2.6)$ & $2(5.3)$ & 0 & $1(2.6)$ & 0 & 0 & 0 \\
\hline S.saprophyticus & 13 & $2(15.4)$ & 0 & 0 & 0 & $5(38.5)$ & 0 & 0 & 0 & 0 & 0 \\
\hline Other CoNS** & 25 & 0 & 0 & 0 & $2(8)$ & 0 & $2(8)$ & $1(4)$ & 0 & 0 & 0 \\
\hline Total $* * *$ & 386 & $16(4.1)$ & $4(1.0)$ & $30(7.8)$ & $95(24.6)$ & 77 (19.9) & $24(6.2)$ & $44(11.4)$ & $5(1.3)$ & $1(0.3)$ & $20(5.2)$ \\
\hline
\end{tabular}

$284 *$ MDR: Multi Drug Resistant, resistant to three or more classes of antimicrobial drugs.

285 ** Other CoNS-species S. caprae, S. warneri, S. condimenti, S. equorum, S. pasteuri, S. salivarius, S. simulans, S. pettenkoferi

$286 * * * 6$ additional isolates (two S. epidermidis, two S. hominis, one S. lugdunensis and one S. pettenkoferi isolate) were omitted from

287 susceptibility testing due to poor growth. 